\title{
GUT and neutrinos
}

\section{Davide Meloni*}

Dipartimento di Matematica e Fisica,

Università degli studi Roma Tre,

INFN, Sezione di Roma Tre

via della Vasca Navale 84, 00146 Roma (Italy)

E-mail: meloniafis.uniroma3. it

In this talk I briefly discuss the present status of models of neutrino mixing with particular emphasis on the concept of Bimaximal Mixing. Two versions of particular GUT models based on $S U(5)$ and on $S O(10)$ are analysed.

Neutrino Oscillation Workshop

4 - 11 September, 2016

Otranto (Lecce, Italy)

${ }^{*}$ Speaker. 


\section{Introduction}

With the continuous improvement of the data, most of the models for neutrino masses and mixing have been discarded by experiment. However, the surviving ones still span a wide range of possibilities ranging from a maximum of symmetry, as those with discrete non-abelian flavour groups to the opposite extreme of Anarchy.

Models based on discrete flavour groups were motivated by the fact that the data seem to suggest some special mixing patterns as good first approximations, like Tri-Bimaximal (TB) or Golden Ratio (GR) or Bi-Maximal (BM) mixing, all having $\sin ^{2} \theta_{23}=1 / 2, \sin ^{2} \theta_{13}=0$ and differing by the value of the solar angle $\sin ^{2} \theta_{12}$. The relatively large $\sin \theta_{13}$, however, has disfavoured TB and GR models because they in general predict too small departures from zero of $\theta_{13}$. Instead in most models of BM the measured value of $\theta_{13} \sim 9^{\circ}$ is more natural. Here I focus on BM mixing [1] which has the following structure:

$$
U_{B M}=\left(\begin{array}{ccc}
\frac{1}{\sqrt{2}} & -\frac{1}{\sqrt{2}} & 0 \\
\frac{1}{2} & \frac{1}{2} & -\frac{1}{\sqrt{2}} \\
\frac{1}{2} & \frac{1}{2} & \frac{1}{\sqrt{2}}
\end{array}\right)
$$

One can consider the possibility that BM is the mixing in the neutrino sector and that the rather large corrective terms to $\theta_{12}$ and $\theta_{13}$ arise from the diagonalization of the charged lepton mass matrix, as obtained in models based on the discrete symmetry $S_{4}[1,2]$. This idea is in agreement with the well-known empirical quark-lepton complementarity relation [3, 4], $\theta_{12}+\theta_{C} \sim \pi / 4$, where $\theta_{C}$ is the Cabibbo angle or, to be less optimistic, with the "weak" complementarity relation $\theta_{12}+$ $\mathscr{O}\left(\theta_{C}\right) \sim \pi / 4$. In addition, the measured value of $\theta_{13}$ is itself of order $\theta_{C}: \theta_{13} \sim \theta_{C} / \sqrt{2}$.

In the following I discuss two examples of GUT models of BM: one based on $S U(5)$ [2] that realizes the program of imposing the $\mathrm{BM}$ structure in the neutrino sector and then correcting it by terms arising from the diagonalization of charged lepton masses; the other based on $S O(10)$ with Type-II see-saw [5], where the origin of BM before diagonalization of charged leptons is left unspecified.

\section{A SUSY $S U(5)$ model with $S_{4}$ discrete symmetry}

This is a variant of the SUSY SU (5) model in 4+1 dimensions [6, 7] with a flavour symmetry $S_{4} \otimes Z_{3} \otimes U(1)_{R} \otimes U(1)_{F N}[1,2]$, where $U(1)_{R}$ implements the R-symmetry while $U(1)_{F N}$ is a Froggatt-Nielsen (FN) symmetry that induces the hierarchies of fermion masses and mixings. The particle assignments are displayed in Tab.1.

As a result of symmetries and field assignments to the ireducible representations of $S U(5) \times S_{4}$, at $\mathrm{LO}$ the charged lepton masses are diagonal and exact BM is realized for neutrinos. Corrections to diagonal charged leptons and to exact BM are induced by vertices of higher dimension in the Lagrangian, suppressed by powers of a large scale $\Lambda$. We adopt the definitions:

$$
\frac{v_{\varphi_{\ell}}}{\Lambda} \sim \frac{v_{\chi}}{\Lambda} \sim \frac{v_{\varphi_{v}}}{\Lambda} \sim \frac{v_{\xi}}{\Lambda} \sim \frac{\langle\theta\rangle}{\Lambda} \sim \frac{\left\langle\theta^{\prime}\right\rangle}{\Lambda} \sim s \equiv \lambda_{C},
$$




\begin{tabular}{|c||c|c|c|c|c|c|c|c|c|c|c|c|c|c|c||}
\hline Field & $F$ & $T_{1}$ & $T_{2}$ & $T_{3}$ & $H_{5}, H_{\overline{5}}$ & $\varphi_{v}$ & $\xi_{v}$ & $\varphi_{\ell}$ & $\chi_{\ell}$ & $\theta$ & $\theta^{\prime}$ & $\varphi_{v}^{0}$ & $\xi_{v}^{0}$ & $\psi_{\ell}^{0}$ & $\chi_{\ell}^{0}$ \\
\hline \hline $\mathrm{SU}(5)$ & $\overline{5}$ & 10 & 10 & 10 & 5,5 & 1 & 1 & 1 & 1 & 1 & 1 & 1 & 1 & 1 & 1 \\
\hline$S_{4}$ & $3_{1}$ & 1 & 1 & 1 & 1 & $3_{1}$ & 1 & $3_{1}$ & $3_{2}$ & 1 & 1 & $3_{1}$ & 1 & 2 & $3_{2}$ \\
\hline$Z_{3}$ & $\omega$ & $\omega$ & 1 & $\omega^{2}$ & $\omega^{2}$ & 1 & 1 & $\omega$ & $\omega$ & 1 & $\omega$ & 1 & 1 & $\omega$ & $\omega$ \\
\hline$U(1)_{R}$ & 1 & 1 & 1 & 1 & 0 & 0 & 0 & 0 & 0 & 0 & 0 & 2 & 2 & 2 & 2 \\
\hline$U(1)_{F N}$ & 0 & 2 & 1 & 0 & 0 & 0 & 0 & 0 & 0 & -1 & -1 & 0 & 0 & 0 & 0 \\
\hline & br & bu & bu & br & bu & br & br & br & br & br & br & br & br & br & br \\
\hline
\end{tabular}

Table 1: Matter assignment of the model. The symbol $\mathrm{br}(\mathrm{bu})$ indicates that the corresponding fields live on the brane (bulk).

where $s=\frac{1}{\sqrt{\pi R \Lambda}}$ is the volume suppression factor, $v_{\phi}$ are the vevs of the flavon fields listed in Tab. 1 and $\lambda_{C}=\sin \theta_{C}$. It turns out that this simple choice leads to a good description of masses and mixings.

\subsection{Charged lepton mass matrices and neutrinos}

For the charged lepton masses we have the following mass matrix:

$$
m_{e} \sim\left(\begin{array}{ccc}
a_{11} \lambda^{5} & a_{21} \lambda^{4} & a_{31} \lambda^{2} \\
a_{12} \lambda^{4} & -c \lambda^{3} & \ldots \ldots \\
a_{13} \lambda^{4} & c \lambda^{3} & a_{33} \lambda
\end{array}\right) \lambda,
$$

where all matrix elements are multiplied by generic coefficients of $\mathscr{O}(1)$. The corresponding lepton rotation is given by:

$$
U_{\ell} \sim\left(\begin{array}{ccc}
1 & u_{12} \lambda & u_{13} \lambda \\
-u_{12}^{*} \lambda & 1 & 0 \\
-u_{13}^{*} \lambda & -u_{12}^{*} u_{13}^{*} \lambda^{2} & 1
\end{array}\right)
$$

$\left(u_{i j}\right.$ again of $\left.\mathscr{O}(1)\right)$ so that $\theta_{23}^{\ell}=0$ in this approximation.

The neutrino sector of the model is unchanged with respect to Ref. [2], so at LO the mass matrix is obtained from the Weinberg operator and the mixing angles are easily derived:

$$
\sin \theta_{13}=\frac{1}{\sqrt{2}}\left|u_{12}-u_{13}\right| \lambda \quad \sin ^{2} \theta_{12}=\frac{1}{2}-\frac{1}{\sqrt{2}} \operatorname{Re}\left(u_{12}+u_{13}\right) \lambda \quad \sin ^{2} \theta_{23}=\frac{1}{2}+\mathscr{O}\left(\lambda^{2}\right) .
$$

We see that, with $\lambda \sim \lambda_{C}$, the model realizes the "weak" complementarity relation and the experimental fact that $\sin \theta_{13}$ is of the same order than the shift of $\sin ^{2} \theta_{12}$ from the BM value of $1 / 2$, both of order $\lambda_{C}$.

\section{Bimaximal mixing in a $S O(10)$ GUT model}

In $S O(10)$ the main added difficulty with respect to $S U(5)$ is that on generation of fermion belongs to the 16-dimensional representation, so that one cannot take advantage of the properties of the $S U(5)$-singlet right-handed neutrinos. A possible strategy to separate charged fermions and 
neutrinos is to assume the dominance of type-II see-saw with respect to the more usual type-I seesaw. In models of this type, the neutrino mass formula becomes $\mathscr{M}_{v} \sim f v_{L}$, where $v_{L}$ is the vev of the $B-L=2$ triplet in the $\overline{\mathbf{1 2 6}}$ Higgs field and $f$ is the Yukawa coupling matrix of the $\mathbf{1 6}$ with the same $\overline{\mathbf{1 2 6}}$.

As one could decide to work in a basis where the matrix $f$ is diagonalised by the TB matrix or by BM matrix, the result of a fit performed in one basis should lead to the same $\chi^{2}$ than the fit in other basis, so the $\chi^{2}$ cannot decide whether TB or BM is a better starting point. Then we need another "variable" to compare whether the data prefer to start from TB or BM. One possibility is to measure the amount of fine-tuning needed to fit a set of data; to this aim, a parameter $d_{F T}$ was introduced in Ref. [5]:

$$
d_{F T}=\sum\left|\frac{\text { pari }_{i}}{e r r_{i}}\right|,
$$

where $e r r_{i}$ is the "error" of a given parameter par $_{i}$ defined as the shift from the best fit value that changes the $\chi^{2}$ by one unit, with all other parameters fixed at their best fit values.

A study of the fine tuning parameter when the fit is repeated with the same data except for $\sin ^{2} \theta_{13}$, which is moved from small to large, shows that the fine tuning increases (decreases) with $\sin \theta_{13}$ for TB (BM) but both BM and TB scenarios are almost equally compatible with the data for similar values of the fine tuning parameter, especially for relatively large $\theta_{13}$.

\section{Summary and Conclusion}

I have discussed two examples of GUT models of BM, one based on $S U(5) \times S_{4}$ and one based on $S O(10)$. In the $S U(5)$ model, the breaking of the $S_{4}$ along the direction $\left\langle\varphi_{v}\right\rangle \sim(0,-1,1)$ imposes the BM structure in the neutrino sector (then corrected by the diagonalization of charged lepton masses) and is useful to implement the weak form of complementarity. In the $S O(10)$ model no clear preference for BM or TB as good LO approximation of the data emerged.

I am very grateful to the organizers of the NOW2016 Workshop for inviting me to present these results and for their kind hospitality.

\section{References}

[1] G. Altarelli, F. Feruglio and L. Merlo, JHEP 0905 (2009) 020.

[2] D. Meloni, JHEP 1110 (2011) 010.

G. Altarelli, P. A. N. Machado and D. Meloni, PoS CORFU 2014 (2015) 012.

[3] M. Raidal, Phys. Rev. Lett. 93 (2004) 161801.

[4] S. Antusch, S. F. King and R. N. Mohapatra, Phys. Lett. B 618 (2005) 150.

[5] G. Altarelli and G. Blankenburg, JHEP 1103 (2011) 133.

[6] E. Witten, Nucl. Phys. B 258 (1985) 75.

Y. Kawamura, Prog. Theor. Phys. 105 (2001) 999.

[7] L. J. Hall and Y. Nomura, Phys. Rev. D 64 (2001) 055003.

Y. Nomura, Phys. Rev. D 65 (2002) 085036.

L. J. Hall and Y. Nomura, Phys. Rev. D 66 (2002) 075004. 